\title{
La sociedad normalizadora en Foucault. A propósito de los sujetos y sujetas al poder
}

Fecha de entrega: 7 de noviembre de 2018

Fecha de evaluación: 18 de diciembre de 2018

Fecha de aprobación: 28 de enero de 2019

Ángela Patricia Rincón Murcia**

\section{Resumen}

En el primer apartado del presente artículo busca evidenciarse la importancia del cuerpo en la práctica política a través del estudio de la antinomia vida-política, que desemboca en la configuración del Estado moderno. En este, el cuerpo individual padece sufrimiento y dolor como resultado de la lógica política de dicho contexto, cuyas estrategias se solidifican mediante las técnicas de poder denominadas anatomopolítica y biopolítica. Posteriormente, se reflexiona sobre el dolor como práctica de dominación política del gobierno neoliberal sobre la corporalidad del sujeto a través de la lógica del hacer vivir dentro de lo que se concibe como el ejercicio de la normalización. Esta última se consigue a través de la vigilancia jerárquica y la sanción normalizadora, que, en oposición a Marx, Foucault no localiza en el

* Este ensayo es un adelanto del proyecto de investigación "Indagaciones en torno al sujeto político en la filosofía actual" elaborado en el marco del semillero Moralopolis del Grupo Interdisciplinario de Investigación en Filosofia y Teología de la Fundación Universitaria San Alfonso. Línea de investigación: filosofia y estudios poscoloniales. Citar como: Rincón Murcia, Á. P. (2019). La sociedad normalizadora en Foucault. A propósito de los sujetos y sujetas al poder. Cuadernos de Filosofía Latinoamericana, 40(121), 95-108. DOI: https://doi.org/10.15332/25005375.5472

** Licenciada en Filosofía y Letras y magíster en Filosofía Latinoamericana de de la Universidad Santo Tomás. Estudiante de doctorado en Filosofía de la Universidad de Antioquia. Líder de Grupo interdisciplinario de Investigación en Filosofía y Teología de la Fundación Universitaria San Alfonso. Investigadora reconocida por Colciencias en la categoría asociada, 2016-2018. Autora de artículos y textos en torno a derechos humanos y filosofía política. Correo electrónico: angieveritatem@gmail.com 
Estado sino en la microfísica de poder. Ello permitirá concluir con la invitación a repensar la anatomopolítica como aspecto fundamental de la política y los sujetos y sujetas que la constituyen.

Palabras clave: normalización, biopolítica, anatomopolítica, corporalidad, política.

\section{Normalizing Society in Foucault. On Subjection to Power}

\section{Abstract}

The first section of the article shows the importance of the body in political practice by addressing the life-politics antinomy, which leads to the configuration of the modern State. Here, the individual body undergoes suffering and pain as a result of that context's political logic, whose strategies are consolidated through the techniques of power known as anatomo-politics and biopolitics. It then carries out a reflection on pain as a neoliberal practice of political domination of the subject's corporeality through the logic of making live, in the context of the exercise of normalization. The latter is achieved through hierarchical surveillance and normalizing sanctions, which, as opposed to Marx, Foucault situates in the microphysics of power rather than in the State. We conclude that it is important to rethink anatomo-politics as a fundamental aspect of politics and of the subjects hat constitute it.

Keywords: normalization, biopolitics, anatomo-politics, corporeality, politics. 


\title{
Introducción
}

\begin{abstract}
El mundo que conocemos no es una figura, simple en suma, en la que todos los sucesos se han borrado para que se acentúen poco a poco los rasgos esenciales, el sentido final, el valor supremo y último; es por el contrario una mirada de sucesos entrecruzados [...]. Creemos que nuestro presente se apoya sobre intenciones profundas, necesidades estables; pedimos a los historiadores que nos convenzan de ello. Pero el verdadero sentido histórico reconoce que vivimos, sin referencias ni coordenadas originarias, en miríadas de sucesos perdidos. (Foucault, 1992, p. 21)
\end{abstract}

Al asumir una investigación en torno al cuerpo como la que se encamina en estas líneas, se hace pertinente recurrir al método foucaultiano que sustenta la correlación entre la historia y el cuerpo como entramado que no puede deshacerse a la hora de interpretar tanto a la sociedad como al individuo, sus juegos de poder y sus espacios otros.

Desde esta perspectiva, se puede citar el siguiente planteamiento de Foucault, quien retoma algunas intempestivas lanzadas por Nietzsche en La gaya ciencia:

Pensamos en todo caso que el cuerpo, por su lado, no tiene más leyes que las de su fisiología y que escapa a la historia. De nuevo error; el cuerpo está aprisionado en una serie de regímenes que lo atraviesan; está roto por los ritmos del trabajo, el reposo y las fiestas; está intoxicado por venenos - alimentos o valores, hábitos alimentarios y leyes morales todo junto; se proporciona resistencias. (Foucault, 1980, p. 19)

A continuación se hilvanan algunos resultados de la presente investigación, sostenida bajo la pregunta de cómo el dominio político del sistema productivo ejerce presión en la vida del sujeto, lo que lleva a su suplicio corporal. Este horizonte de sentido permite asumir una mirada analítica desde la microfísica del poder, en oposición a la salida marxista de abolir el Estado como base de las ideologías de opresión.

Como se desarrollará en las líneas posteriores, la mirada foucaultiana permite encontrar los ejercicios milimétricos del poder desde el régimen de la disciplina, que no se limita al Estado de derecho, sino que por el contrario se ejerce desde las prácticas concretas de la normalización. 


\section{La antinomia vida-política}

Quizá lo que hoy se requiera, al menos para quien hace de la filosofía su profesión, sea el camino inverso: no tanto pensar la vida en función de la política, sino pensar la política en la forma misma de la vida.

(Esposito, 2011, p. 22)

En su obra Bios. Biopolítica y filosofía, Roberto Esposito retoma los planteamientos de Foucault sobre el concepto de biopolítica. Evidencia allí cómo para el autor de $\mathrm{La}$ voluntad de saber la biopolítica no simplemente se constituye por la indagación en torno al modo en que la vida orienta y determina la política, sino ante todo por la manera en que la vida resulta afectada, invadida, aferrada, desafiada, penetrada por la política (Esposito, 2011, p. 51).

Esta transformación conceptual establecida por Foucault contiene el problema que se produce por la tensión entre las categorías de vida y política, el cual se reproduce como una antinomia constante. En el marco de dicha antinomia distingue Foucault dos tipos de técnicas, a saber: la biopolítica y la anatomopolítica, cuyos contenidos se describen a continuación.

\section{La anatomopolítica: poder sobre el cuerpo}

Este apartado se orienta a delinear la constitución del cuerpo como referente en la conformación del Estado moderno y la correspondiente interpretación foucaultiana de dicha máquina de poder.

Al reinterpretar la base conceptual de los promulgados derechos a la vida y a la libertad, teorizados en el periodo moderno del contractualismo, Foucault evidencia el surgimiento de la técnica anatomopolítica. Esta empieza a ejercerse sobre el hombrecuerpo, cuya configuración moderna se enmarca dentro de lo que puede denominarse hombre-máquina.

Es decir, en respuesta a la configuración civil del Estado, bajo la insignia de la protección individual, se requiere un poder igual de individualizante con el objetivo de aprovechar dichas fuerzas particulares en aras de la productividad económica del Estado y 
a la luz de su progreso. Los instrumentos de los que se vale esta técnica de poder para alcanzar tal fin los encontramos a través de los diferentes dispositivos disciplinarios que se configuran a la luz de la praxis gubernamental, tales como la escuela o el ejército:

Concretamente, ese poder sobre la vida se desarrolló desde el siglo Xvir en dos formas principales; no son antitéticas; más bien constituyen dos polos de desarrollo enlazados por todo un haz intermedio de relaciones. Uno de los polos, al parecer el primero en formarse, fue centrado en el cuerpo como máquina: su educación, el aumento de sus aptitudes, el arrancamiento de sus fuerzas, el crecimiento paralelo de su utilidad y su docilidad, su integración en sistemas de control eficaces y económicos, todo ello quedó asegurado por procedimientos de poder característicos de las disciplinas: anatomopolítica del cuerpo humano. (Foucault, 2007b, p. 168)

El segundo polo de desarrollo del poder al que se refiere Foucault es la biopolítica, que será abordada en el siguiente apartado. Por su parte, la anatomopolítica se orienta, como lo referencia el autor, a la docilización de los cuerpos, constituyéndose en una tecnología individualizante del poder. Su objetivo es producir cuerpos fragmentados y dóciles, orientados hacia el rendimiento productivo.

En este contexto la antinomia entre vida y política que se conceptualizó al inicio del presente texto hace que cada una de dichas categorías se torne fuerza yuxtapuesta de la otra. Tal tensión se manifiesta a través del dominio que la segunda ejerce en la primera, por medio de la docilización de los cuerpos, lo cual en últimas desemboca en la constitución de un cuerpo enajenado para el sistema orgánico estatal, al que debe orientar sus fuerzas en aras de la producción y el progreso. Una política que busca hacer vivir al cuerpo productivo para solidificar el andamiaje del sistema, lo que lleva consecuentemente a dejar morir el cuerpo que no produce, bajo delicadas e invisibles estrategias que inmunizan de cualquier señalamiento a dicha práctica gubernamental.

Es así como el ejercicio de poder estatal configura una estructura cuidadosamente organizada en cada una de sus partes, en aras del sostenimiento de la utilidad, la cual sienta sus bases en la relación inseparable entre derecho individual y mercado. De esta manera, cada una de las leyes y estructuras de poder ha de responder al sostenimiento de la mencionada relación. 
Para cerrar este apartado cabe recalcar que el dispositivo anatomopolítico del poder gubernamental constituye una fuente de estudio en la biopolítica de Foucault, quien en La voluntad de saber, publicada en 1975, opone este concepto al de soberanía. A su vez, en ese mismo año Foucault ofrece su lección titulada “Defender la sociedad”, en donde establece la distinción que ya se mencionó entre las dos tecnologías de poder, considerando que la anatomopolítica hace parte de la biopolítica, en otras palabras, esta engloba a aquella.

\section{La biopolítica: incidencia del dominio político en la vida biológica}

Esta tecnología del poder, posterior a la anatomopolítica se constituye con el fin de orientar las poblaciones humanas hacia el objetivo político que se desee. En este sentido, ya no se remite al individuo y su cuerpo, sino que específicamente aplica su dispositivo sobre la población y sus cuestiones biológicas y políticas. Al respecto, en Defender la sociedad, Foucault argumenta:

Luego de la anatomopolítica del cuerpo humano, introducida durante el siglo XVIII, vemos aparecer, a finales de este, algo que ya no es esa anatomopolítica, sino lo que yo llamaría una biopolítica de la especie humana. ¿Cuál es el interés central en esa nueva tecnología del poder, esa biopolítica, ese biopoder que está estableciéndose? [...] Se trata de un conjunto de procesos como la proporción de los nacimientos y las defunciones, la tasa de reproducción, la fecundidad de una población, etcétera. (Foucault, 2003, p. 208)

Desde este horizonte argumentativo, considera el autor que en la segunda mitad del siglo XVIII la acción médica adquiere una connotación de saber supremo encargado de ejecutar el proceso de la normalización. Así, en una acción medicalizadora de la población, se busca incidir en lo que se va a concebir como una higiene pública. De este modo, la medicina se constituye en el saber por antonomasia en la revisión de los diferentes procesos de constitución de la sociedad, interviniendo en los escenarios de la reproducción, la natalidad y la morbilidad.

Con respecto a esta última, aclara Foucault que en el momento de la aparición de la biopolítica lo que más interesa al saber médico ya no van a ser las epidemias típicas de la época medieval. Estas se caracterizaban por el drama del riesgo constante de 
una muerte inminente para todos, por lo cual se consideraba necesario establecer un proceso curativo de exclusión. Tal como lo evidencia el autor con respecto al leproso en su texto Los anormales, en donde hace una genealogía de la concepción de la lepra como epidemia reinante:

La exclusión de la lepra era una práctica social que implicaba, en principio, una partición rigurosa, una puesta a distancia, una regla de no contacto entre un individuo (o grupo de individuos) y otro. Se trataba por una parte de la expulsión de esos individuos hacia un mundo exterior, confuso, más allá de las murallas de la ciudad, más allá de los límites de la comunidad. Constitución por consiguiente de dos masas ajenas una a la otra. Y la que era echada, lo era en sentido estricto hacia las tinieblas exteriores. Por último, [...] esta exclusión del leproso implicaba la descalificación — tal vez no exactamente moral, pero en todo caso sí jurídica y política - de los individuos expulsados. (Foucault, 2007a, pp. 50-51)

En la coyuntura de la biopolítica, en cambio, la función se orienta a atender las endemias, esto es, la tipificación de las enfermedades que más se propagan en la población, en especial cuando estas interfieren en el proceso de producción del individuo. Máxime si se tiene en cuenta que la conformación del sistema económico capital hace del sujeto un homo faber por antonomasia antes de su constitución como ciudadano. Por ello es necesario que el saber médico se oriente a definir el tiempo de duración de dichas enfermedades, su patología, así como la consecuente incapacitación del enfermo.

De este modo, dicho saber médico se orienta a tipificar:

Enfermedades más o menos difíciles de extirpar y que no se consideran, como las epidemias, en concepto de causas de muerte más frecuente sino como factores permanentes - y así se las trata - de sustracción de fuerzas, disminución del tiempo de trabajo, reducción de las energías, costos económicos, tanto por lo que deja de producirse como por los cuidados que pueden requerir. En suma, la enfermedad como fenómeno de población: ya no como la muerte que se abate brutalmente sobre la vida - la epidemia - sino como la muerte permanente, que se desliza en la vida, la carcome constantemente, la disminuye y la debilita. (Foucault, 2003, p. 209) 
A partir de esta orientación del saber enmarcado en la industrialización puede verse, siguiendo al autor, que la biopolítica también buscaría intervenir en los fenómenos de tipo incapacitante, que ocurren bien sea por accidentalidad o por vejez. La estrategia de dicha intervención consiste en promover en el individuo la convicción de lo imperante que es su ejercicio de producción, en tanto que la economía generada por su trabajo le va a servir para sostenerse en caso de que entre en el marco de dichos fenómenos de invalidez. Esta realidad lo obligan a aumentar su producción en tanto tenga las condiciones físicas para ello pues dicha productividad le permitirá establecer un ahorro para su sobrevivencia en las mencionadas situaciones. Así, desde el saber productivo, unido al saber médico, se establece la noción de una autoprotección preventiva, que reemplazaría el denominado asistencialismo gubernamental, el cual constituye una garantía que frena, según el saber médico mercantilista, el afán productivo del individuo.

Esta argumentación foucaultiana se opone a la visión de Marx sobre la lucha de clases y la abolición del Estado. Foucault define a esta última como el postulado de la localización, que ubica el uso del poder en el aparato estatal. Al respecto, el autor de $\mathrm{La}$ microfísica del poder argumenta que este no tiene una localización en un ente específico, de manera que sería una apuesta equivocada la pretensión marxista de tomarse el poder aboliendo el Estado ya que el poder es un ejercicio milimétrico y difuso.

Dentro de dicha lógica milimétrica las dos técnicas del biopoder que se han expuesto (anatomopolítica y biopolítica) no implican yuxtaposición, sino que, por el contrario, se complementan en los dominios del cuerpo individual y de la población:

\footnotetext{
Estos dos poderes no constituirían, como a veces se argumenta, dos teorías en el pensamiento de Foucault, excluyentes, independientes y sucesivas una de otra, sino más bien dos modos conjuntos de funcionamiento del saber/poder, aunque, es cierto, con focos, puntos de aplicación, finalidades y apuestas específicas: el adiestramiento de los cuerpos, por una parte; la regulación de la población, por la otra. (Fontana y Bertani, 2003, p. 238)
}

Cerrando esta argumentación en torno a las dos técnicas del biopoder, se hace necesario argüir que - pese a que en El nacimiento de la biopolítica Foucault se concentra en el concepto de gubernamentalidad dejando de lado los de biopolítica y anatomopolítica por considerar que aquel responde de manera más amplia al concepto de poblaciónesta ponencia se ha enfocado en el contenido conceptual de la anatomopolítica. En 
particular se orienta hacia la posibilidad de una comprensión del sufrimiento del cuerpo, que se constituye en territorio de castigo del ritual político en el que se instaura el poder a través de diversos dispositivos de presión.

\section{Dolor corpóreo y presión política}

Como se ha mencionado, el dolor infligido al sujeto por la dominación política constituye una temática ineludible en la actual reflexión filosófica. Es por ello que para Roberto Esposito una de las tareas fundamentales de la filosofía consiste en ampliar los problemas de la política, de manera que esta disciplina no se reduzca al tema de la dominación, sino que dirija la mirada a sus implicaciones en los diferentes ámbitos de la persona.

Precisamente, una de las mencionadas implicaciones se manifiesta en el dolor como práctica de dominación política sobre la corporalidad del sujeto. En ese sentido, en el marco de la biopolítica se presenta la preocupación por la relación entre política y vida biológica, que expresa lo real en su más concreta manifestación: el sufrimiento corporal.

Así, afirma Esposito, "nunca como en estos días, la política se practicó sobre los cuerpos y en los cuerpos de víctimas inermes e inocentes" (2006, p. 16). Por ello, se ha considerado pertinente investigar en torno a la deriva biopolítica, en la cual se sitúa específicamente la anatomopolítica como un análisis filosófico acerca de la perversión en los cuerpos producida por los sistemas políticos concebidos en el pensamiento contemporáneo.

Dicho bosquejo filosófico ha buscado orientar sus cauces por las investigaciones que hiciera Michel Foucault, quien en su estudio acerca del concepto de neoliberalismo nos brinda una base teórica de comprensión sobre cómo funcionan el control y el dominio de los ciudadanos mediante el suplicio de los cuerpos.

Así, en su análisis sobre la Escuela de Chicago Foucault la define como una radicalización del ordoliberalismo alemán, que ejerce el dominio a través de una mercantilización de la vida misma, incluso de la vida íntima, de modo que las necesidades básicas, como la salud, simplemente son inversiones que el individuo debe adelantar para competir en el juego de la economía. 
En este punto resulta pertinente la afirmación de Santiago Castro en su estudio sobre Foucault, titulado Historia de la gubernamentalidad: "El mercado puede hacer vivir a unos, pero, del mismo modo, puede hacer morir a otros, concretamente a aquellos que no pueden o no quieren ser 'empresarios de sí mismos”' (Castro, 2010, p. 211).

Hacer vivir constituye así el lema de la etapa anatomopolítica, en la medida en que el camino del "triunfo" se orienta hacia la producción económica, que contiene las bases del Estado sostenido en el ideal de progreso y la estrategia de la docilización de los cuerpos:

Muera todo lo sólido, viva todo lo que carece de memoria y se deja llevar por el fluido permanente de la innovación y el consumo. Limpieza social "políticamente correcta", en tanto que se realiza a través del mercado y no del Estado, pero no por ello menos dramática e inquietante. (Castro, 2010, p. 212)

En efecto, el cuerpo dócil, el cuerpo de cada individuo, de cada ciudadano, permanece fácilmente en el juego del progreso, en la medida en que, dominado por la función automatizadora de este, aquel deja de ser su dueño: su función en el juego siempre ha de apuntar hacia la consolidación del progreso, establecido en términos económicos como aumento de la producción.

\section{A modo de conclusión: una invitación a repensar la anatomopolítica}

En coherencia con el método investigativo que se dibuja en la introducción de este texto, enfocado hacia la problematización del cuerpo, resulta pertinente abordar este apartado conclusivo con el siguiente cuestionamiento: ¿Continúa de modo incesante e irremediable la enajenación del cuerpo al sistema productivo? La respuesta es negativa. El desenfreno del denominado juego del progreso no tarda en manifestar sus efectos perversos.

Para explicarlo, de acuerdo con el horizonte corporal que se ha sostenido a lo largo de estas líneas se puede pensar al Estado como cuerpo compuesto por sus docilizadas partes. En el marco del cuerpo estatal aparece, pues, una tensión en respuesta a que se 
ataca a sí mismo atentando contra las partes que lo componen. Así, aquellos cuerpos que ha buscado docilizar evidencian la tensión entre las fuerzas en lucha.

Aquí se puede acudir a Roberto Esposito, quien a partir del concepto de inmunización refiere que en dicho proceso tanatopolítico se busca "condicionar la potenciación de la vida a la consumación cada vez más extendida de la muerte" (2011, p. 18). Y continúa Esposito: "No porque resulte extraño a la lógica inmunitaria, sino, al contrario, porque es parte de ella de manera paroxística, hasta el punto de dirigir sus dispositivos protectores contra su propio cuerpo, tal como sucede en las enfermedades autoinmunes" (p. 19).

¿Cómo se soluciona la problemática que genera ese cuerpo individual que debido a un proceso estatal de autoinmunización de la lógica neoliberal resulta desengranado de la máquina del poder productivo? Ello es lo que permite dar respuesta negativa al interrogante anteriormente mencionado - ¿Continúa de modo incesante e irremediable la enajenación de dicho cuerpo? - . Es en el momento de esta tensión cuando la lógica anatomopolítica devuelve al individuo automatizado el cuerpo que se le había apropiado.

Así, el improductivo cuerpo que genera tensión en la corporeidad estatal pierde su valor, y en tanto minusvalor se convierte en una problemática individual, personal, que se establece como responsabilidad del sujeto. Este es el momento en el que, como se menciona líneas arriba, el individuo debe haber obtenido las ganancias básicas de su producción para proveerse de los medios económicos que le permitan sostener su poco productiva vida antes de tener la discreción de fallecer.

De este modo el cuerpo, cuya praxis se entrega durante toda su historia a contribuir a los modelos de producción del sistema que lo trasciende, es devuelto al sujeto desde el momento en que no aporta productivamente en la lógica de mercado del sistema anatomopolítico. Siguiendo con el esquema de dicho dispositivo del biopoder: son a esos cuerpos a los que hay que eliminar. Los precarios sistemas de salud propios de la lógica neoliberal del mercado son simplemente una evidencia de ello ya que sustentan el esquema milimétricamente concebido por dicha lógica, bajo la cual hay que dejar morir al sujeto improductivo, acto que se hace bajo la capa de la inmunidad proveída por la ley que sustenta la producción de dicho sistema. 
De esta manera, el padecimiento del cuerpo deja de ser el suplicio público que se concebía en el siglo xvi europeo para consolidarse como un castigo, una apropiación más discreta de la fuerza física del individuo que aporta al andamiaje del sistema.

Al respecto puede citarse la siguiente situación, enunciada por Foucault en Vigilar $y$ castigar, en torno a un condenado a muerte en 1757 por parricidio (asesinar a su padre):

Damiens fue $[. .$.$] "llevado y conducido en una carreta, desnudo, en camisa,$ con un hacha de cera encendida de dos liras de peso en la mano"; después, "en dicha carreta, a la plaza de Gréve, y sobre un cadalso que allí habría sido levantado (deberán serle) atenaceadas las tetillas, brazos, muslos y pantorrillas, y su mano derecha, asido en esta el cuchillo con que cometió dicho parricidio, quemada con fuego de azufre, y sobre las partes atenaceadas se le verterá plomo derretido, aceite hirviendo, pez resina ardiente, cera y azufre fundidos juntamente, y a continuación, su cuerpo estirado y desmembrado por cuatro caballos y sus miembros y tronco consumidos en el fuego, reducidos a cenizas y sus cenizas arrojadas al viento". (2009, p. 11; la interpolación entre paréntesis está en el original)

Este modelo de castigo público, cuya función es evidenciar el suplicio al cuerpo como tránsito a una muerte que impone el rey, hace ver al ente gubernamental como una expresión de jerarquía que produce miedo. La transición humanista moderna quiso atemperar dicha imagen de crueldad del Estado para hacerlo ver como un conducto regulador de los derechos del individuo y su protección, un constructo civil humanizado (concebido contractualmente), que logra cambiar la lógica de hacer morir al desobediente por el esquema de hacer vivir al individuo productivo. Ha de tenerse en cuenta que a este se lo ha convencido de su rol como productor de un sistema, tarea en la que con creces ayuda la educación con su cada vez mayor énfasis en la formación para la productividad por encima de la formación de la humanidad. El castigo corporal actual se traduce, dentro de esta transformación gubernamental, en una actividad silenciosa consistente en la docilización del cuerpo, al que se le exige enmarcarse en los esquemas de lo normal según el dominio neoliberal.

Todo este esquema propende hacia la visibilización de una supuesta humanización del entramado estatal en aras de establecer la confianza que, en torno al progreso, debe 
generar a los ciudadanos, más allá de la pretendida humanización de los individuos. Sin embargo, se transfigura ahora un sistema que prefiere el suplicio discreto, deja solo al enfermo y permite que muera silenciosamente, con lo cual disimula su crueldad ante el cuerpo sufriente. En la voz de Foucault, nos encontramos ahora ante

unos castigos menos inmediatamente físicos, cierta discreción en el arte de hacer sufrir, un juego de olores más sutiles, más silenciosos, y despojados de su fasto visible [...]. El castigo ha cesado poco a poco de ser teatro. Y todo lo que podía llevar consigo de espectáculo se encontrará en adelante afectado de un índice negativo. (2009, pp. 15-16)

Este es entonces el proceso constitutivo del suplicio de los cuerpos, que se configuró en la lógica conceptual del cuerpo-máquina a través de un poder individualizante que arranca las fuerzas del ciudadano-cuerpo para volverlo dócil, y por tanto, útil. Evidenciando esto, en Defender la sociedad, Foucault muestra que en el marco de la modernidad la vida adquiere niveles de sentido cuando es fuente de producción que requiere ser potenciada tanto para el dominio de la población como para el afianzamiento del progreso capitalista.

Como se había mencionado en líneas anteriores, la anatomopolítica es un conjunto de procedimientos orientados a la producción de individuos, o mejor, de cuerpos individuales que a la vez que se hacen útiles se docilizan. El detalle es la preocupación esencial de este conjunto de procedimientos: hay que establecer en cada cuerpo, en cada individuo concreto, los obstáculos que enfrenta, las posibles formas de resistencia que opone y el modo de neutralizarlas, las regularidades que sigue y que debe seguir de acuerdo con finalidades específicas. Para esto se debe atender no solamente a las grandes variaciones del comportamiento, sino también, y sobre todo, a las que parecen irrelevantes, incluso a las que pasan inadvertidas en el ámbito privado por excelencia: la familia.

Este bosquejo conceptual resalta la necesidad de indagar en torno al cuerpo y su suplicio. En efecto, hay que analizar la condición de sujeción de los individuos en el marco de la vigilancia jerárquica, que reproduce a sujetos y sujetas de un poder disciplinar dispuesto a sancionarles con base en los criterios de normalización que enmarcan el saber y el discurso. 


\section{Referencias}

Castro, S. (2010). Historia de la gubernamentalidad I. Razón de Estado, liberalismo y neoliberalismo en Michel Foucault. Bogotá: Siglo del Hombre.

Esposito, R. (2006). Biopolítica y filosofía. Buenos Aires: Grama.

Esposito, R. (2011). Bíos. Biopolítica y filosofía. Buenos Aires/Madrid: Amorrortu.

Fontana, A. y Bertani, M. (2003). Situaciones que tienen los individuos. En M. Hay que defender la sociedad (pp. 217-238). Madrid: Akal.

Foucault, M. (1980). Microfísica del poder. Madrid: La Piqueta.

Foucault, M. (1992). Verdad y poder. En Microfísica del poder (pp. 175-189). Madrid: La Piqueta.

Foucault, M. (2003). Hay que defender la sociedad. Madrid: Akal.

Foucault, M. (2007a). Los anormales. México: FCE.

Foucault, M. (2007b). Historia de la sexualidad I. La voluntad de saber. México: Siglo XXI.

Foucault, M. (2009). Vigilar y castigar. Nacimiento de la prisión. México: Siglo XXI. 